\title{
Non-Lie Symmetry Groups and New Exact Solutions to the $(2+1)$-Dimensional Broer-Kaup System
}

\author{
Bin Zheng ${ }^{1}$, Xiqiang Liu $^{2}$ \\ ${ }^{1}$ School of Mathematics and Physics, Southwest University of Science and Technology, Mianyang, China \\ ${ }^{2}$ School of Mathematics Sciences, Liaocheng University, Liaocheng, China \\ Email: zbinlisa@sina.com
}

Received July 17, 2012; revised September 21, 2012; accepted October 1, 2012

\begin{abstract}
For the (2 + 1)-dimensional Broer-Kaup system, we study the corresponding Lie symmetry groups, and obtain the symmetry group theorem and the Backlund transformation formula of solutions finding. At the same time, we find some new exact solutions of the (2+1)-dimensional Broer-Kaup system and extend the results in the papers [1-4].
\end{abstract}

Keywords: Broer-Kaup System; Lie Symmetry Group; Exact Solution; Backlund Transform

\section{Introduction}

Many non-linear phenomena, such as the non-linear waves in fluid mechanics, the laser phenomenon in non-linear optics and the non-linear behaviors in the field of plasma, can be described by the non-linear partial differential equations (systems). Therefore quite naturally, solving these non-linear partial differential equations (systems) and articulating the characteristics of the solutions become an important topic of investigation for a large number of mathematicians and physicists. In paper [5], by use of symmetry constraint in the reduction of the Kadomtsev-Petviashvili equation we obtain the following $(2+1)$-dimensional Broer-Kaup (BK) system:

$$
\begin{aligned}
& H_{t y}+2 G_{x x}+2\left(H H_{x}\right)_{y}-H_{x x y}=0 \\
& G_{t}+2(G H)_{x}+G_{x x}=0
\end{aligned}
$$

Thence studies by a number of scholars appeared, investigating this system. Paper [6] discusses the system's Painleve characteristic and that it possesses infinite symmetrical problems of arbitrary time variable $t$ and space variable $y$, and reaffirms that system (1) is an integrable system. Paper [1] uses the extended homogeneous balance method and separation of variables to discuss the localized coherent structure of system (1). Paper [2] by using the Lie-group optimized system classifies the solutions of system (1) and furthermore finds some new explicit solutions. Through the application of the extended homogeneous balance method, papers $[3,4]$ obtain some exact solutions to system (1) and explore the system's induced phenomenon. Paper [3,7] in particular discusses the application of advanced BK equations in extensive fields of studies such as non-linear optical fiber commu- nication and fluid mechanics.

Lie-group method is a powerful tool in the investigation of non-linear partial differential equations (systems) [8]. Using classical or non-classical Lie-symmetry method we are able to obtain a large number of solutions to the non-linear evolution equations (systems) (see papers [912]). Especially put forward are the direct reduction method in paper [9] and the more recent direct method in papers [11,12], which provide a simple and direct method to investigate the non-Lie symmetry groups of non-linear evolution equations. In this paper, we are going to use the direct reduction method to discuss the Lie point symmetry group and non-Lie symmetry group of the $(2+$ 1)-dimensional integrable BK system (1). In doing that we obtain the system's corresponding symmetry group theorem and the Backlund transformation formula of solutions finding, through which we are able to obtain some new exact solutions to the BK system. We therefore extend the results in paper [1-4].

\section{Lie Point Symmetry Group}

In order to simplify the calculation, we make the transformation $G=H_{y}$, substituting it into system (1) we obtain:

$$
H_{y t}+2 H_{x} H_{y}+2 H H_{x y}+H_{x x y}=0
$$

As a result, we transform the discussion of BK system (1) into the investigation of Equation (2). Suppose Equation (2) has the following form of solution:

$$
H(x, y, t)=\alpha(x, y, t)+\beta(x, y, t) U(\xi, \eta, \tau)
$$

in which $\xi=\xi(x, y, t), \quad \eta=\eta(x, y, t), \quad \tau=\tau(x, y, t)$ 
are undetermined functions. Also, function $U(\xi, \eta, \tau)$ of its independent variables is required to satisfy similar Equation (2), that is, to satisfy the following equation:

$$
U_{\xi \xi \eta}=-U_{\eta \tau}-2 U_{\xi} U_{\eta}-2 U U_{\xi \eta}
$$

Now the prime task is to determine the undetermined functions in Equation (3). Substitute Equations (3) and (4) into Equation (2), and in the meanwhile let the coefficients of the partial derivatives in $U(\xi, \eta, \tau)$ be zero. We are able to obtain the following system:

$$
\begin{aligned}
& \xi_{y}=0, \eta_{x}=0, \tau_{x}=0, \tau_{y}=0, \beta_{y}=0, \eta_{t}=0, \\
& \xi_{x}^{2}=\tau_{t}, \beta=\xi_{x}, \xi_{t}+2 \beta \alpha=0, \beta_{t}+2 \beta \alpha_{x}=0,
\end{aligned}
$$

Solve the above over-determined equations we obtain the following results:

$$
\begin{gathered}
\tau=\tau(t), \eta=\eta(y), \xi=\delta x \tau^{\prime \frac{1}{2}}+F_{0}(t), \\
\beta=\delta \tau^{\prime \frac{1}{2}}, \alpha=-\frac{\delta x \tau^{\prime \prime}+2 F_{0}^{\prime} \tau^{\prime \frac{1}{2}}}{4 \delta \tau^{\prime}},
\end{gathered}
$$

where $\delta^{2}=1$ and $\tau(t), \eta(y), F_{0}(t)$ are arbitrary smooth functions.

From above we can sum up and arrive at the main conclusion of this paper, that is:

Theorem: if $U=U(\xi, \eta, \tau)$ is a solution to Equation (4), then

$$
H=-\frac{\delta x \tau^{\prime \prime}+2 F_{0}^{\prime} \tau^{\prime \frac{1}{2}}}{4 \delta \tau^{\prime}}+\delta \tau^{\prime \frac{1}{2}} U(\xi, \eta, \tau), G=H_{y}
$$

is also a solution to Equation (1), in which $\delta^{2}=1$ and $\tau(t), \eta(y), F_{0}(t)$ are arbitrary smooth functions determined by Equations (5) and (6).

From the derived theorem we know that the symmetry group of Equation (2) is constituted by two parts; one is the Lie point symmetry group $\mathfrak{R}$ when $\delta=1$; the other the Lie point symmetry group $\mathfrak{R}$ reflection Lie group of $x$ when $\delta=-1$, which is obtained by the Lie point symmetry transformation $\{x, U\} \rightarrow\{-x,-U\}$. In order to analyze and compare the obtained Lie point symmetry group of Equation (2) and the result obtained from standard symmetry group method, we derive the following from Equations (5) and (6):

$$
\tau(t)=t+\varepsilon f(t), \eta(y)=\varepsilon g(y), F_{0}(t)=\varepsilon F(t), \delta=1
$$

here $\varepsilon$ an infinitesimal, $f(t), g(y), F(t)$ are arbitrary functions. Therefore from Equation (7) we can obtain:

$$
\begin{gathered}
H=U+\varepsilon \sigma(U), \\
\sigma(U)=-\frac{1}{4} x f^{\prime \prime}(t)-2 F^{\prime}(t)+\frac{1}{2} f^{\prime} U \\
+\left(\frac{x}{2} f^{\prime}(t)+F(t)\right) U_{x}+g(y) U_{y}+f(t) U_{t},
\end{gathered}
$$

in which $\sigma(U)$ is the symmetry of Equation (2), its corresponding group generator (vector field) is:

$$
\begin{aligned}
V= & \left\{\frac{x}{2} f^{\prime}(t) \frac{\partial}{\partial x}+f(t) \frac{\partial}{\partial t}+\left(-\frac{x}{4} f^{\prime \prime}(t)+\frac{1}{2} f^{\prime} U\right) \frac{\partial}{\partial U}\right\} \\
& +g(y) \frac{\partial}{\partial y}+\left\{F(t) \frac{\partial}{\partial x}-\frac{1}{2} F^{\prime}(t) \frac{\partial}{\partial U}\right\} \\
= & V_{1}(f(t))+V_{2}(g(y))+V_{3}(F(t))
\end{aligned}
$$

where

$$
\begin{aligned}
& V_{1}=\frac{x}{2} f^{\prime}(t) \frac{\partial}{\partial x}+f(t) \frac{\partial}{\partial t}+\left(-\frac{x}{4} f^{\prime \prime}(t)+\frac{1}{2} f^{\prime} U\right) \frac{\partial}{\partial U}, \\
& V_{3}=F(t) \frac{\partial}{\partial x}-\frac{1}{2} F^{\prime}(t) \frac{\partial}{\partial U}, \\
& V_{2}=g(y) \frac{\partial}{\partial y},
\end{aligned}
$$

From calculation we know that the generator (8) of the Lie point symmetry group is the same as the result obtained from standard Lie group method, here we are not going to discuss in details. However, what needs to be pointed out is that the corresponding transformation group determined by Equation (7) has become non-Lie point symmetry group.

\section{Exact Solutions to the Broer-Kaup System}

We can see from the established symmetry group theorem that we have already obtained the Backlund transformation formula of finding solutions (7) for system (1). By taking some known solutions from the Broer-Kaupequation as seed solutions, we are able to find numerous new exact solutions to the BK system by use of Equation (7). The form of such solutions is as following:

$$
\begin{aligned}
H(x, y, t)= & -\frac{\delta x f^{\prime \prime}(t)-2 f^{\prime}(t) F^{\prime}(t)}{4 \delta f^{\prime}(t)} \\
& +\delta f^{\frac{1}{2}}(t) U(\xi, \eta, \tau) \\
G(x, y, t)= & H_{y},
\end{aligned}
$$

here $\xi=\delta x f^{\prime}(t)+F(t), \quad \eta=g(y), \quad \tau=f(t), \quad \delta^{2}=1$. $U(\xi, \eta, \tau)$ is the equation's solution of arbitrary form. Next we are going to demonstrate the application of the formula. Take the solution of the following form from paper [4]:

$$
\begin{aligned}
& H(x, y, t)=P(y)+\frac{\sum_{k=1}^{n} v_{k} a_{k} \exp \left(a_{k} x-a_{k}^{2} t-2 P a_{k} t+b_{k}\right)}{v_{0}+\sum_{k=1}^{n} v_{k} \exp \left(a_{k} x-a_{k}^{2} t-2 P a_{k} t\right)} \\
& G(x, y, t)=H_{y}
\end{aligned}
$$


where $v_{k}=v_{k}(y), a_{k}=a_{k}(y), b_{k}=b_{k}(y)$. When $n=1$, $v_{0}=v_{1}=$ const , $P=$ const , we are enabled to obtain the soliton solution of the BK equation:

$$
\begin{aligned}
H(x, y, t)= & P(y)+2^{-1} a_{1} \\
& +2^{-1} a_{1} \tanh \left(2^{-1}\left(a_{1} x-a_{1}^{2} t-2 P(y) a_{1} t+b_{1}\right)\right) \\
G(x, y, t)= & H_{y},
\end{aligned}
$$

Substitute $U(\xi, \eta, \tau)$ in (9) with Equation (10) as a seed solution, in which $\xi=\delta x f^{\prime}(t)+F(t), \eta=g(y)$, $\tau=f(t), \delta^{2}=1$. Then we are able to obtain a new solution to Equation (9) as following:

$$
\begin{aligned}
H(x, y, t)= & -\frac{\delta x f^{\prime \prime}(t)-2 f^{\prime}(t) F^{\prime}(t)}{4 \delta f^{\prime}(t)}+\delta f^{\frac{1}{2}}(t) P(y) \\
& +2^{-1} a_{1}+2^{-1} a_{1} \tanh \left(2 ^ { - 1 } \left(a_{1} \delta x f^{\prime}(t)+F(t)\right.\right. \\
& \left.\left.-a_{1}^{2} f(t)-2 P(y) a_{1} f(t)+b_{1}\right)\right) \\
G(x, y, t)= & H_{y}
\end{aligned}
$$

Figure 1 is shown for us to observe more accurately the evolution characteristic of solution (11). When $P=0$, $a_{1}=2, b_{1}=2 y^{2}, t=3, f(t)=3 t^{3}, F(t)=0, \delta=1$, we respectively obtain solutions $H$ and $G$ which have shapes (a) and (b) determined by Equation (10) and shapes (c) and (d) by Equation (11).

Figure 2 shows that when $P=0, a_{1}=2 y, b_{1}=2 y^{2}$, $t=3, f(t)=3 t^{3}, \quad F(t)=0$. The solutions $H$ and $G$ have shapes (a) and (b) that are determined by Equation (10) and shapes (c) and (d) by Equation (11). Compared to (10), Equation (11) contains some arbitrary functions; in addition, the velocity and amplitude of the soliton change according to the changes of the independent variables in such functions, thus the structure of the new soliton solution, that is, Equation (11) has more remarkable regional changing characteristic.

If we take other solutions in papers [3-6] as seed solutions, by the same token we will be able to obtain corresponding new exact solutions to BK system (1) by the use of Equation (7); in this paper we are not going to list them out. As a conclusion, regarding the exact solutions to equation system (1), we extend the results of paper [3-6].

\section{Conclusion}

This paper through application of the simple direct reduction method discusses the Lie point symmetry group and non-Lie symmetry group of the $(2+1)$-dimensional Broer-Kaup system (1) and obtains the Backlund transformation formula of solutions finding. Helped by the main theorem at which we arrived, we are enabled to

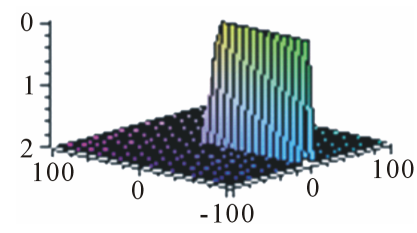

(a)

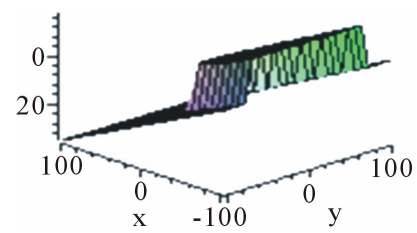

(c)

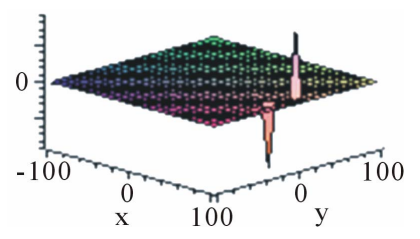

(b)

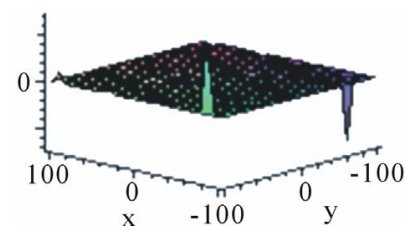

(d)
Figure 1. $P=1, a_{1}=2, b_{1}=2 y^{2}, t=3, f(t)=3 t^{3}, F(t)=0, \delta=$ 1.

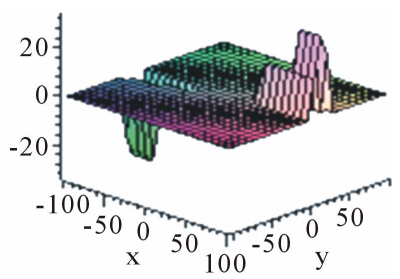

(a)

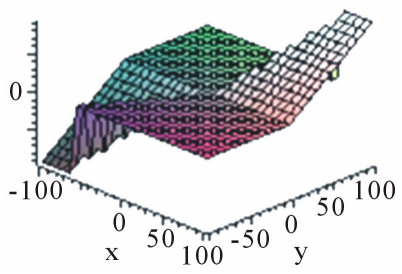

(c)

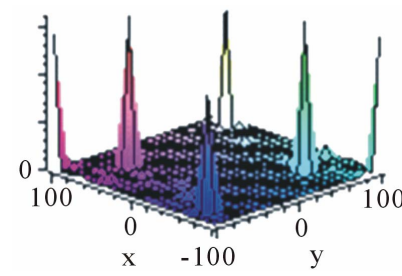

(b)

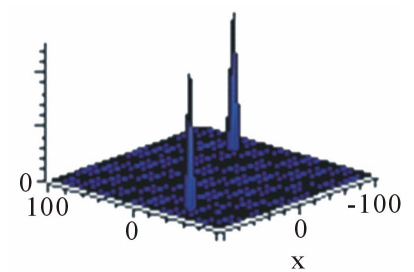

(d)
Figure 2. $P=1, a_{1}=2 y, b_{1}=2 y^{2}, t=3, f(t)=3 t^{3}, F(t)=0, \delta=$ 1.

generate a large number of new exact solutions to the BK system. From the figures given we can see that although there is no substantial change in the overall structure of the solutions, the regional changing characteristic in the new solutions is obviously more remarkable.

\section{Acknowledgements}

This paper was completed during the visit at Liaocheng University; the author (Zheng Bin) would like to sincerely thank the Faculty of Mathematics of Liaocheng University for providing generous assistant.

\section{REFERENCES}

[1] J. F. Zhang and P. Han, "The Regional Structure of (2 + 1)-Dimensional Broer-Kaup Equation,” Physics Journal, Vol. 51, 2002, pp. 705-711.

[2] X. Q. Liu, "New Explicit Solutions to the $2+1$ Dimensional Broer-Kaup Equations,” Journal of Partial Differ- 
ential Equations, Vol. 17, 2004, pp. 1-11.

[3] C. L. Bai, X. Q. Liu, C. J. Bai and B. Z. Xu, "Multiple Soliton Solutions of the $2+1$ Dimensional Broer-Kaup Equations,” Acta Photonica Sinica, Vol. 28, 1999, pp. 10291031.

[4] C. L. Bai, X. Q. Liu and H. Zhao, "New Localized Excitations in a $(2+1)$ Dimensional Broer-Kaup System," Chinese Physics, Vol. 14, No. 2, 2005, pp. 285-292. doi:10.1088/1009-1963/14/2/012

[5] S. Y. Lou and X. B. Hu, "Broer-Kaup System from Dar Boux Transformation Related Symmetry Constraints of Kadomtsev-Petviashvili Equation,” Communications in Theoretical Physics, Vol. 29, No. 1, 1998, pp. 145-148.

[6] H. Y. Ruan and Y. X. Chen. Acta Physica Sinica, Vol. 7, 1998, p. 241.

[7] D. J. Huang and H. Q. Zhang, "Variable Coefficient Projective Riccati Equation Method and Its Application to a New $2+1$ Dimensional Simplified Generalized BroerKaup System,” Chaos, Solitons and Fractals, Vol. 23, No. 2, 2005, pp. 601-607. doi:10.1016/j.chaos.2004.05.011
[8] P. J. Olver, “Applications of Lie Groups to Differential Equations,” Springer-Verlag, New York, 1993. doi:10.1007/978-1-4612-4350-2

[9] P. A. Clarkson, "Nonclassical Symmetry Reduction of the Boussinesq Equation,” Chaos, Solitons and Fractals, Vol. 5, No. 12, 1995, pp. 2261-2301. doi:10.1016/0960-0779(94)E0099-B

[10] P. A. Clarkson and M. D. Kruskal, "New Similarity Solutions of the Boussinesq Equation," Journal of Mathematical Physics, Vol. 30, No. 10, 1989, pp. 2201-2213. doi:10.1063/1.528613

[11] S. Y. Lou and H. C. Ma, "Non-Lie Symmetry Groups of $2+1$ Dimensional Nonlinear Systems Obtained from a Simple Direct Method," Journal of Physics A: Mathematical and General, Vol. 38, No. 7, 2005, pp. L129-L137. doi:10.1088/0305-4470/38/7/L04

[12] H. C. Ma, “A Simple Method to General Lie Point Symmetry Groups of the $3+1$ Dimensional Jimbo-Miwa Equation,” Chinese Physics Letters, Vol. 22, No. 3, 2005, pp. 554-557. doi:10.1088/0256-307X/22/3/010 